\title{
Identification of GROWTH-REGULATING FACTOR transcription factors in lettuce (Lactuca sativa) genome and functional analysis of LsaGRF5 in leaf size regulation
}

Bin Zhang ${ }^{1,2,3+}$, Yanan Tong ${ }^{4 \dagger}$, Kangsheng Luo ${ }^{4}$, Zhaodong Zhai ${ }^{5}$, Xue Liu ${ }^{1,2,3}$, Zhenying Shi ${ }^{6}$, Dechun Zhang ${ }^{4^{*}}$ and Dayong Li, $\mathrm{ir}^{1,2,3^{*}}$

\begin{abstract}
Background: GROWTH-REGULATING FACTORs (GRFs), a type of plant-specific transcription factors, play important roles in regulating plant growth and development. Although GRF gene family has been identified in various plant species, a genome-wide analysis of this family in lettuce (Lactuca sativa L.) has not been reported yet.

Results: Here we identified 15 GRF genes in lettuce and performed comprehensive analysis of them, including chromosomal locations, gene structures, and conserved motifs. Through phylogenic analysis, we divided LsaGRFs into six groups. Transactivation assays and subcellular localization of LsaGRF5 showed that this protein is likely to act as a transcriptional factor in the cell nucleus. Furthermore, transgenic lettuce lines overexpressing LsaGRF5 exhibited larger leaves, while smaller leaves were observed in LsaMIR396a overexpression lines, in which LsaGRF5 was down-regulated.

Conclusions: These results in lettuce provide insight into the molecular mechanism of GRF gene family in regulating leaf growth and development and foundational information for genetic improvement of the lettuce variations specialized in leaf character.
\end{abstract}

Keywords: Lettuce, GROWTH-REGULATING FACTOR, Leaf development, MicroRNA396, Genome-wide analysis

\section{Background}

As one of the plant-specific transcription factors, GROWTH-REGULATING FACTORs (GRFs) are important regulators in plant growth and development. The first identified GRF functions on gibberellic acid (GA)induced stem elongation [1]. In various studies, the functions of GRF genes were found in development of

\footnotetext{
*Correspondence: zhangdc227@163.com; lidayong@nercv.org ${ }^{\dagger}$ Bin Zhang and Yanan Tong contributed equally to this work. ${ }^{1}$ National Engineering Research Center for Vegetables, Beijing Vegetable Research Center, Beijing Academy of Agriculture and Forestry Science, Beijing 100097, PR China

${ }^{4}$ Biotechnology Research Center, China Three Gorges University, Yichang 443002, PR China

Full list of author information is available at the end of the article
}

leaf, stem, seed and root by regulating cell proliferation or cell expansion to form large organs [1-8], other new functions in flowering, stress response and plant longevity were uncovered recently [9-15]. Two conserved domains, the QLQ (Gln, Leu, Gln, InterPro: IPR014978, PFAM: PF08880) domain, which is considered to be a protein-protein interaction domain, and WRC (Trp, Arg, Cys, InterPro: IPR014977, PFAM: PF08879) domain, which is supposed to be involved in DNA binding, are found in the N-terminal of GRF family proteins $[1,2,8$, 16-18].

GRF family genes are found to be involved in plant growth, development and regeneration. The seedlings with overexpressed miR396-resistant AtGRF1 or AtGRF3 
showed shorter roots [9]. BnGRF2 could increase seed weight and oil content by upregulating the expression of chloroplast-related genes in rapeseed (Brassica napus) [19]. In rice (Oryza sativa), OsGRF4 played an important role in grain weight [20,21]. GRF proteins form functional transcriptional complex with the transcription cofactor GRF-Interacting Factors (GIF) [22], and GRF-GIF chimeras could dramatically boost regeneration in various species [23]. Furthermore, most Arabidopsis thaliana GRF genes play important roles in leaf size control [6-8]. Among the nine AtGRF genes, six GRF genes, AtGRF1, AtGRF2, AtGRF3, AtGRF4, AtGRF5 and $A t G R F 9$, were proved to function in leaf development $[3,4,12]$. Overexpression of AtGRF1 and AtGRF2 respectively cause larger leaves with increased cell size, while atgrf1/2/3 triple mutant showed smaller and narrower leaves [2]. Overexpression of AtGRF5 exhibit bigger leaves due to increased cell number but not cell size [3]. The function of AtGRF5 could not be replaced by other AtGRFs, though some functions of them overlap $[3,12]$. AtGRF9 contribute to determining final leaf size, although it has a minor role in cell proliferation [3, 24].

miRNAs, about 20 nucleotides (nt) in length, are single-strand, non-coding, small-molecular-weight RNAs, which could regulate gene expression through target mRNA cleavage or/and translational inhibition [25-27]. Genome-wide analyses reveal that GRFs and a few bZIP transcription factor genes are the major targets of microRNA396 (miR396) [28]. miR396 shares nearly perfect sequence complementarity with the transcript of WRC motif in seven members of the AtGRF genes, except for AtGRF5 and AtGRF6 in Arabidopsis thaliana [29, 30]. Correspondingly, miR396a and miR396b regulate leaf growth and development by repressing the expression of AtGRFs [29, 30]. MiR396a regulates flower formation, including sepal-petal identity, by regulating the expression of GRF gene [15]. Additionally, the miR396-AtGRF module could also regulate adaxial-abaxial (Ad-Ab) polarity formation during leaf morphogenesis [31].

The ever-developing whole-genome sequencing technology, identifies GRF genes in various plant species, such as Arabidopsis thaliana [2], Oryza sativa [16], Zea mays [32], Brassica rapa [33], Solanum lycopersicum [34], Pyrus bretschneideri, Vitis vinifera [35], Brassica napus [36], Nicotiana tabacum [37] and Populus trichocarpa [38]. Lettuce (Lactuca sativa L.) is an important leafy vegetable, the leaf size of which has significant meaning for production. The whole genome sequence of lettuce cultivar 'Salinas' has been recently released [39]. However, the GRF gene family in lettuce has not been evaluated yet. In this study, we identified 15 GRF genes in lettuce and respectively named them based on their chromosomal location. They were divided into six groups according to phylogenic tree of LsaGRFs and AtGRFs. To investigate the transcriptional factor character, LsaGRF5 was specifically studied for its subcellular location and transcriptional activity. In addition, LsaGRF5 was found to be cleaved as a target of miR396. Using transgenic plants, LsaGRF5 could enhance the leaf growth in lettuce and Arabidopsis thaliana when overexpressed, while smaller leaves were obtained through overexpression of Lsa-miR396 in lettuce. These results may provide a foundation for further elucidation of the function of LsaGRFs and Lsa-miR396 in leaf growth regulation in lettuce.

\section{Results}

\section{Identification of the GRF genes and miR396s in lettuce}

To identify the GRF gene family in lettuce, the GRF genes from Arabidopsis thaliana, rice and tomato were firstly used as the query sequences for BLASTN searching in lettuce genome database. Thus, thirteen candidate GRF genes were obtained in lettuce. GRF protein contains two conserved functional domains, QLQ and WRC. Therefore, QLQ and WRC were used for the Hidden Markov Model (HMM) search, and another two candidate $L s a G R F$ genes were identified. Finally, the amino acids sequences of all fifteen candidate LsaGRF genes were applied for BLASTP searching, and no further hits were got. Thus, there are totally $15 \mathrm{Lsa}$ $G R F$ genes identified in lettuce genome. Based on their respective location on the chromosomes, we designated them as LsaGRF1 to LsaGRF15 respectively (Fig. 1A). LsaGRFs were distributed on each chromosome of lettuce, except for chromosome 1 and 7, and the most (five LsaGRFs) occurred on chromosome 6 (Fig. 1A). The basic information of LsaGRF1-15, including gene ID, chromosomal location, length of gene and protein, PI value and exon numbers, was listed in Table 1. Most LsaGRFs had 3 or 4 exons, while LsaGRF4 and LsaGRF9 had 2 exons and LsaGRF7 had 6 exons (Table 1). In addition, the predicted isoelectric point $(\mathrm{pI})$ values of LsaGRF preoteins were between 6 and 9, except that the pI values of LsaGRF4 and LsaGRF7 were higher than LsaGRF9 (Table 1). The amino acid sequence alignment of 15 LasGRF was showed in Fig. S1. All of the 15 LsaGRFs contained the conserved WRC domain, but only 13 LsaGRFs had complete QLQ domain. There is no QLQ domain in LsaGRF4, and LsaGRF7 has only an incomplete QLQ domain (Fig. 1B).

The GRF genes are known as the target of miR396s. To date, two MIR396s in Arabidopsis (Ath-MIR396s) and seven MIR396s in rice (Osa-MIR396s) are identified. There are five MIR396s, MIR396a to MIR396e, in lettuce genome [39]. The phylogenic trees of five LsaMIR396s and MIR396s in lettuce, Arabidopsis and rice were shown in Fig. S2A and B, respectively, based 


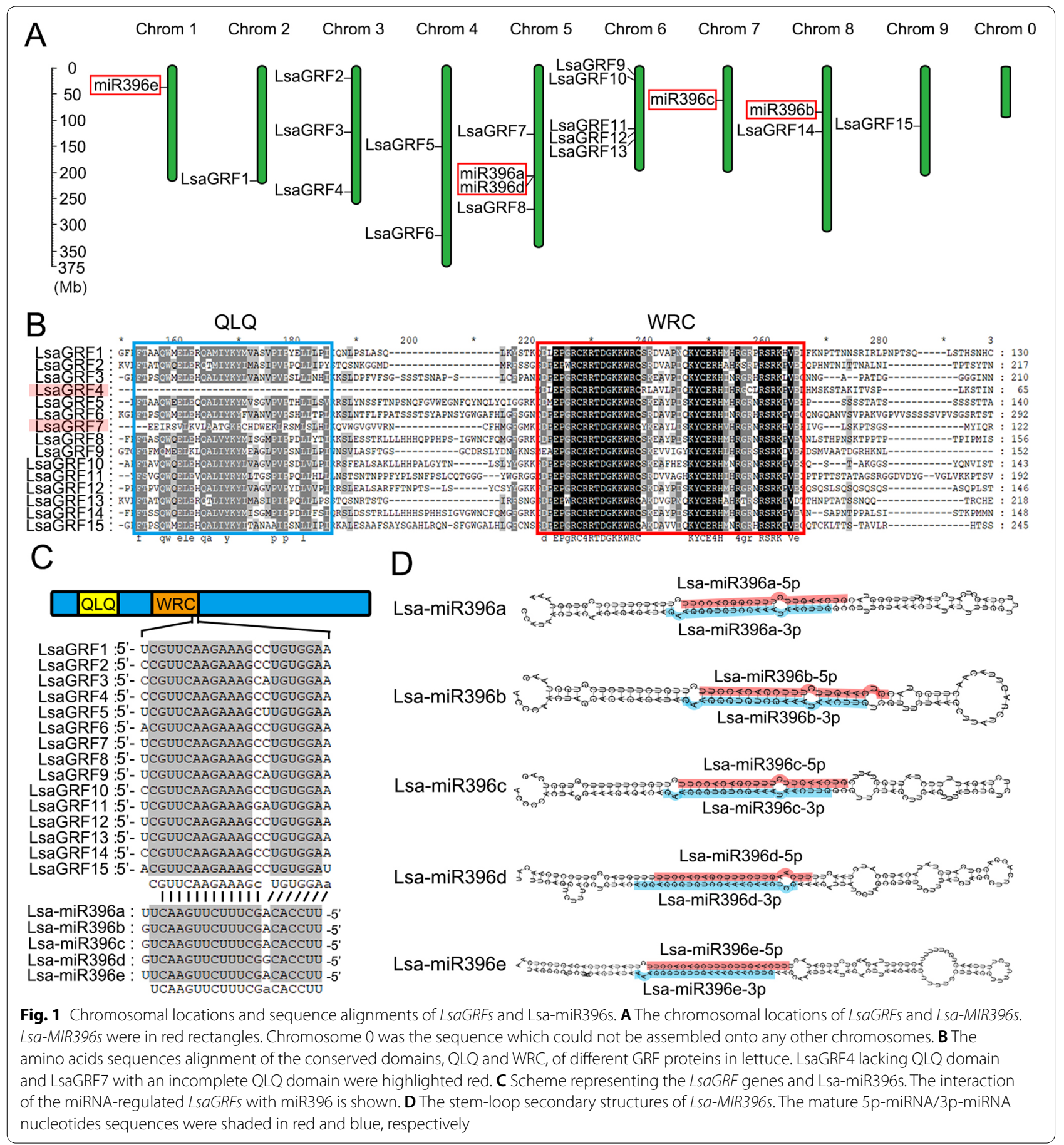

on their stem-loop sequences. The target sequence of miR396s locates at the end of the WRC domain (Fig. 1C). Although the stem-loop structures were totally distinct, five Lsa-miR396s were highly conserved in the mature region with only two nucleotides difference (Fig. 1C and D). Five Lsa-MIR396s were located on chromosome 1, 5, 7 and 8 respectively, among which chromosome 5 contained two Lsa-MIR396s, LsaMIR396a and Lsa-MIR396d (Fig. 1A). The identified GRF genes and miR396s in major species were listed and compared with those in lettuce (Table S1). The number of $L s a G R F$ genes was the second largest, and the number of miR396s was comparable to that in rice and tomato. 
Table 1 Characteristics of the GRFs in lettuce

\begin{tabular}{|c|c|c|c|c|c|c|c|}
\hline Name & Accession No. & Chr & CDS (bp) & Exon No. & Length (aa) & MW (KDa) & $\mathrm{pl}$ \\
\hline LsaGRF1 & Lsat_1_v5_gn_2_135541.1 & Chr02 & 990 & 3 & 329 & 36.06 & 8.76 \\
\hline LsaGRF2 & Lsat_1_v5_gn_3_14660.1 & Chr03 & 1227 & 4 & 408 & 44.51 & 6.24 \\
\hline LsaGRF3 & Lsat_1_v5_gn_3_85521.1 & Chr03 & 999 & 4 & 332 & 37.74 & 8.73 \\
\hline LsaGRF4 & Lsat_1_v5_gn_3_133500.1 & Chr03 & 702 & 2 & 233 & 27.17 & 9.63 \\
\hline LsaGRF5 & Lsat_1_v5_gn_4_92941.1 & Chr04 & 951 & 3 & 316 & 36.58 & 8.81 \\
\hline LsaGRF6 & Lsat_1_v5_gn_4_159961.1 & Chr04 & 1551 & 4 & 516 & 56.20 & 7.70 \\
\hline LsaGRF7 & Lsat_1_v5_gn_5_54781.3 & Chr05 & 603 & 6 & 200 & 22.70 & 9.27 \\
\hline LsaGRF8 & Lsat_1_v5_gn_5_141200.1 & Chr05 & 990 & 3 & 329 & 37.54 & 8.24 \\
\hline LsaGRF9 & Lsat_1_v5_gn_6_10680.1 & Chr06 & 459 & 2 & 152 & 16.89 & 8.66 \\
\hline LsaGRF10 & Lsat_1_v5_gn_6_17460.1 & Chr06 & 897 & 3 & 298 & 33.12 & 6.44 \\
\hline LsaGRF11 & Lsat_1_v5_gn_6_70601.1 & Chr06 & 1113 & 4 & 370 & 40.08 & 8.23 \\
\hline LsaGRF12 & Lsat_1_v5_gn_6_75441.1 & Chr06 & 1014 & 3 & 337 & 37.60 & 8.74 \\
\hline LsaGRF13 & Lsat_1_v5_gn_6_81681.1 & Chr06 & 1029 & 4 & 342 & 36.78 & 7.09 \\
\hline LsaGRF14 & Lsat_1_v5_gn_8_86361.1 & Chr08 & 1071 & 3 & 356 & 40.63 & 8.97 \\
\hline LsaGRF15 & Lsat_1_v5_gn_9_78540.1 & Chro9 & 1233 & 4 & 410 & 44.10 & 7.67 \\
\hline
\end{tabular}

\section{Phylogenetic analysis, gene structures and motif divergence of LsaGRFs}

To explore the phylogenic relationship of GRF gene family in different species, phylogenetic analysis, intron-exon and motif characteristics of LsaGRFs were performed. Phylogenetic analysis of GRF family in lettuce was firstly assessed and visualized using a Neighbor-Joining phylogenetic tree (Fig. 2A). All of the 15 LsaGRFs were divided into two groups (including 9 and 6 LsaGRFs respectively), each of which contained two small subgroups in the phylogenetic tree. Gene structure and motifs were considered to have a divergence during gene evolution. Therefore, the gene structures and motifs were listed in phylogenetic tree's order (Fig. 2B). Most LsaGRF genes contain three or four exons. The LsaGRF genes containing the same number of exons were in the same group. For examples, LsaGRF4 and LsaGRF9 both have two exons. LsaGRF11, 1, 2 and 13 have four exons and the rests in another group have three exons expect $L s a G R F 7$
(Fig. 2B). In conserved motifs analysis, motif 1 (yellow rectangle) and motif 2 (purple rectangle) were present to be the WRC and QLQ protein domain. As shown in Fig. 2B, the LsaGRF proteins in the same branch of phylogenetic tree have similar position and numbers of QLQ and WRC domains. All these analyses showed that the phylogenic relationship of LsaGRF genes was highly consistent with the gene structures and motif divergence of $G R F$ genes in lettuce.

The functions of GRF genes in Arabidopsis and rice were extensively studied. The phylogenic relationship of GRF genes in lettuce, Arabidopsis and rice were helpful for putative function prediction of GRF genes in lettuce. There are totally 12 and 9 GRF genes in rice and Arabidopsis, respectively. The Neighbor-Joining phylogenetic tree of GRF genes from lettuce, rice and Arabidopsis showed that there were six groups according to the tree (Fig. 3). It was revealed that the phylogenic relationship of GRF genes in lettuce, rice and Arabidopsis
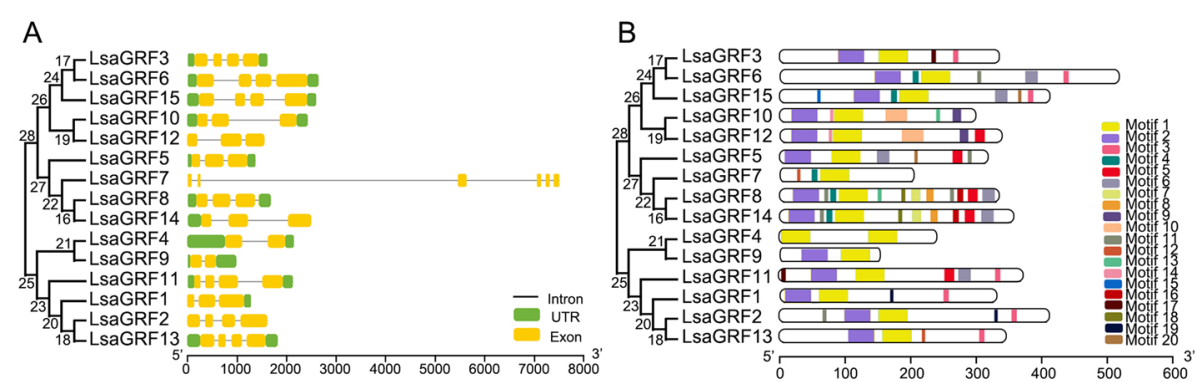

Fig. 2 Gene structures and motif composition of LsaGRFs. A The gene structures of LsaGRFs. B The motif composition of LsaGRF proteins with MEME. Twenty motifs are represented by different colored boxes. The same color rectangle in different proteins referred to the same motif. The sizes of motifs are proportional to their sequence lengths. The order of $L$ saGRF genes were based on the phylogenetic tree shown on the left of the figures. The size scales of gene and protein length were indicated at the bottom 
were divergence. There were two groups, group I and VII, harboring GRF genes from lettuce, rice and Arabidopsis, which indicated that these LsaGRFs had putative orthologous genes in both rice and Arabidopsis. In group II and IV, there were just LsaGRFs and AtGRFs, but no $G R F$ genes from rice, while there was no GRF gene from Arabidopsis in group VI. Group III contained only two LsaGRFs, LsaGRF4 and LsaGRF9, but no AtGRFs or OsGRFs, while group V contained three OsGRFs and one
AtGRF gene, but no LsaGRF gene. Interestingly, the role of AtGRF5 could not be taken over by other members of AtGRFs, though there were partly overlapping functions between AtGRFs [3, 12]. Therefore, we chose the putative homolog gene of AtGRF5, LsaGRF5, based on the phylogenic relationship derived from phylogenic tree for further functional analysis (Fig. 3).

Phylogenetic tree was constructed for 15 Lactuca sativa, 9 Arabidopsis thaliana and 12 Oryza sativa

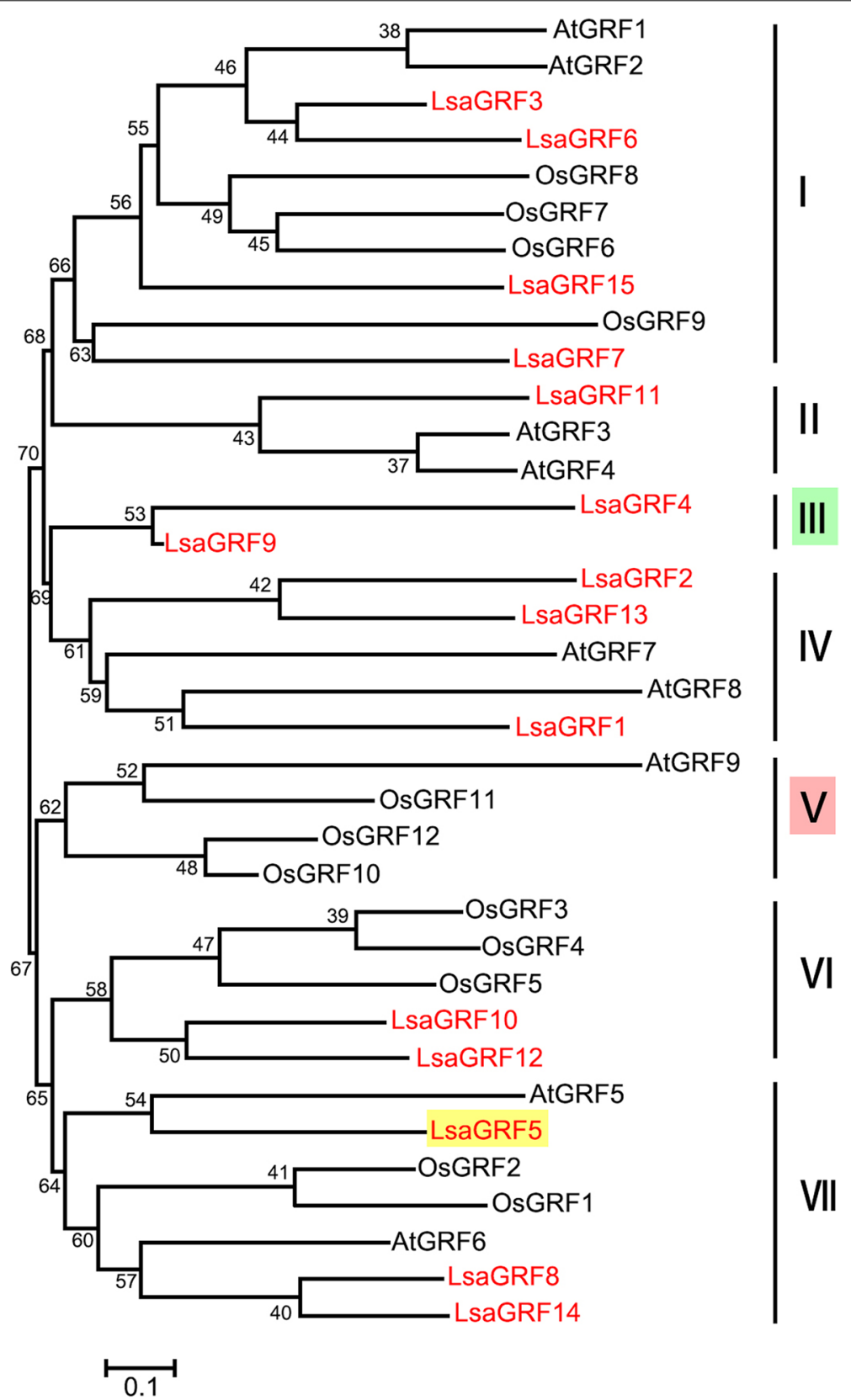

Fig. 3 Phylogenetic relationship between GRFs from Arabidopsis, rice and lettuce 


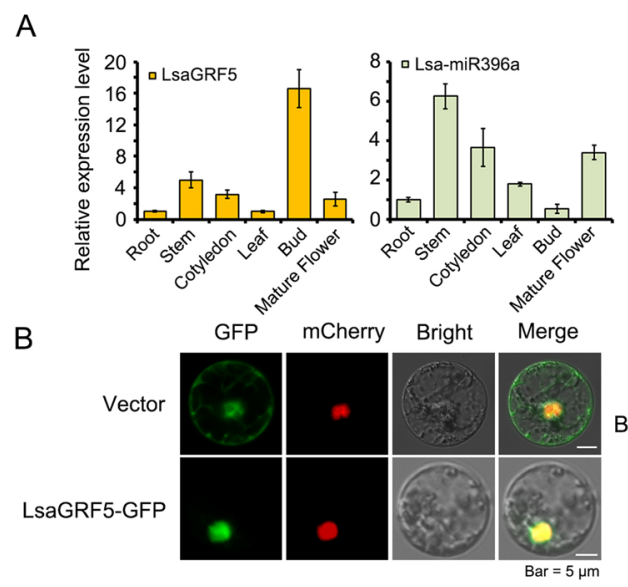

C
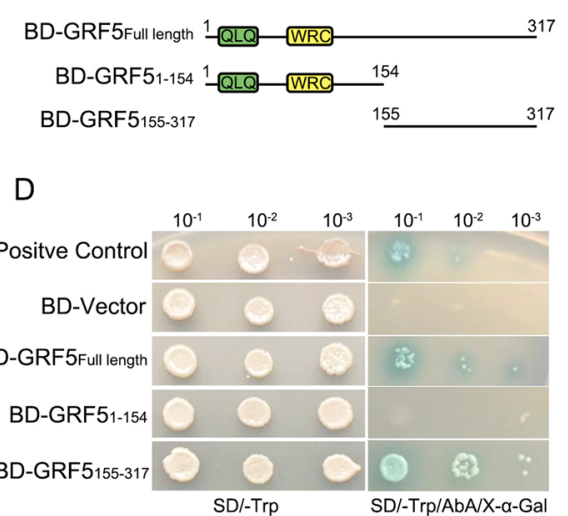

Fig. 4 The functional characterization of $L S a G R F 5$ gene and characterization of LsaGRF5 protein. A The expression profiles of $L s a G R F 5$ and pri-Lsa-MIR396a in different tissues of the lettuce'YDL' Actin was used as a reference gene. B Subcellular localization of LsaGRF5 in lettuce protoplasts. Confocal images showed that the fluorescence of LsaGRF5-GFP fusion protein was completely overlapped with that of Ghd7-mCherry, which was specifically expressed in the nucleus. The vector with 355::Ghd7-mCherry only was used as a control. Bar $=5 \mathrm{~mm}$. C The schematic diagrams of the constructions used in transactivation assay in yeast. The full-length, N-terminal region (1-154 aa) containing QLQ and WRC domains and C-terminal region (155-317 aa) of the LsasGRF5 were respectively fused into DNA sequences containing a GAL4 DNA-binding domain in pGBKT7 (BD). D Transactivation assay of different LsaGRF5 constructs in yeast. The constructs in (B) were expressed in the yeast strain Y2HGold with PGBDT7-OsMYB103L as a positive control and the negative control plastid (pGBKT7). The transformants with different diluted concentrates were dropped on the SD/-trp and SD/-trp/AbA/X-alpha-gal plates. After 2-4 days at $30^{\circ} \mathrm{C}$, possible transcriptional activation functions of LsaGRF5-full length and LsaGRF5 ${ }_{155-317}$ were observed

GRF proteins. There were 7 phylogenetic clusters designated as I-VII. LsaGRFs were written with red fronts. The cluster III that contained GRFs only from lettuce was highlighted with green and the cluster $\mathrm{V}$ without LsaGRFs was shaded in red. The LsaGRF5, which was selected for further investigation, were highlighted in yellow. The scale bar represents 0.1 amino acid changes per site.

\section{Functional characterization of LsaGRF5}

To characterize the putative functions of LsaGRF5, the expression profile of $L s a G R F 5$ was detected. From the quantitative real-time PCR (qRT-PCR) results, the expression levels of $L s a G R F 5$ in roots and leaves were relatively low, while those in the bud were significantly high (Fig. 4A). However, in mature flowers with mature pollens and pistils, the expression level of LsaGRF5 was much lower comparing that in the bud (Fig. 4A), indicating that LsaGRF5 probably function in flower development. Meanwhile, we also detected the expressions of $L s a-m i R 396 a$, putatively regulating the expression of GRF genes, in these tissues. We found that Lsa-miR396a were relatively highly expressed in stem, cotyledon and mature flower, while significantly much lower in buds (Fig. 4A). The tissues with high Lsa-miR396a expression, e.g. mature flowers and stems, showed relatively low expression of LsaGRF5. And vice versa the tissues with high LsaGRF5 expression, e.g. buds, showed relatively low expression of $L s a-m i R 396 a$., indicating that $L s a G R F 5$ might be regulated by Lsa-miR396a.

The LsaGRF proteins are putative TFs. We chose the LsaGRF5 and performed its subcellular location observation and transactivation assay. We isolated the protoplast cell of lettuce and transformed the vector containing 35S:LsaGRF5-GFP and 35S:Ghd7-mCherry, which was reported to locate in nuclear, into lettuce protoplast cells. The empty vector was used for control. As shown in Fig. 4B, green fluorescence of GFP and red fluorescence of mCherry were totally overlapped in the protoplast cell transformed by 35S:LsaGRF5-GFP, indicating that the LsaGRF5-GFP and Ghd7-mCherry have the same nuclear localization. While the protoplast cell transformed by empty vector exhibited ubiquitous green fluorescence, excepting the overlapped region with the red nuclear fluorescence of Ghd7-mCherry (Fig. 4B). Therefore, LsaGRF5 located in the nucleus.

To identify which part of the LsaGRF5 protein had the transcriptional activity, we divided the LsaGRF5 into two parts based on the conserved protein domains. One part is the N-terminal of LsaGRF5, GRF5 ${ }_{1-154}$, containing QLQ and WRC domains, and the other, GRF5 ${ }_{155-317}$ (Fig. 4C). Full-length and two partial LsaGRF5s were constructed into yeast expression vector, $p G B D-T 7$. The empty vector $p G B D-T 7$ and $p G B D-T 7-O s M Y B 103 L$ which was proved to have transcriptional activity were designed as the negative and positive control, respectively 
[40]. These recombinant plasmids were transformed into yeast strain Y2HGold. They showed similar growth states without Tryptophan (Trp) under different diluted concentration (Fig. 4D), indicating that the recombinant plasmids were indeed transformed into the yeast cells and the transformation made few influences on the yeast growth. The yeast cells expressed full-length GRF5 and GRF $_{155-317}$ could grow with AbA (Aureobasidin A) and turn blue with $\mathrm{X}$-alpha-galactoside, which were the same as the positive control (Fig. 4D). These results suggested that the $\mathrm{C}$-terminal contributed to the transcriptional activity of LsaGRF5, while the $\mathrm{N}$-terminal containing QLQ and WRC domains did not.

\section{LsaGRF5 is a miR396a target gene in lettuce}

The GRF gene family is known as the target of miR396 $[29,30]$. To verify this in lettuce, we firstly predicted the complementarity between Lsa-miR396 and LsaGRFs. Lsa-miR396a shared nearly perfect complementarity with 14 LsaGRFs except LsaGRF9 (Fig. S3). The free energies of duplex structures were all lower than - $30 \mathrm{kcal} / \mathrm{mol}$ except for $L s a G R F 9$ ( $-30.6 \mathrm{kcal} / \mathrm{mol}$ ) (Fig. S3). It means that all $L s a G R F$ genes except for $L s a G R F 9$ probably were the targets of Lsa-miR396a. We chose LsaGRF5 for further verification, and performed the $5^{\prime}$ RNA ligase-mediated (RLM) rapid amplification of cDNA ends (RACE) assay. The results showed that the $1 \sim 10$ bp of the target sequence in LsaGRF5 did not exist in the sequencing results, which means the transcript of $L s a G R F 5$ was cleaved at base 10 of the miR396 target site (Fig. 5). Therefore, LsaGRF5 was probably the target of Lsa-miR396a. Function analysis in vivo could further clarify the regulatory relationship between $L s a G R F 5$ and Lsa-miR396.

\section{The phenotypes of LsaGRF5 and Lsa-miR396 overexpression lines}

To investigate the function of $L s a G R F 5$, we constructed the overexpression lines in lettuce. LsaGRF5 driven by CaMV 35S promoter was transformed into the lettuce cultivar of Romaine type 'YIDALI' (YDL). Eleven independent transgenic lines were obtained. Transformation verification was carried out through a pair of primers located on $35 \mathrm{~S}$ promoter and LsaGRF5, respectively. The results showed that five out of eleven were positive transgenic lines with the same band as the positive control (Fig. 6A). qRT-PCR assay revealed that the expression of LsaGRF5 increased $5 \sim 15$ folds in these five lines (Fig. 6B). Two lines, LsaGRF5-OE5 and LsaGRF5-OE11, with high LsaGRF5 expressions were used for further phenotypic analysis. The leaves of LsaGRF5-OE5 and LsaGRF5-OE11 were significantly bigger than these of YDL transformed by empty vector (OE-) (Fig. 6C). The bigger leaves also existed in LsaGRF5 overexpressed in Arabidopsis (Fig. S4). From these results, LsaGRF5 could enhance the leaf growth, and this function was conserved in Arabidopsis.

To figure out the functional relevance between LsamiR396a and LsaGRF5, we also overexpressed LsaMIR396a in YDL. Three positive transgenic lines were obtained by PCR of genomic DNA (Fig. 6A). The transcriptional levels of Lsa-miR396a were about $8 \sim 15$ times higher in overexpression lines compared with negative control (Fig. 6B). Two overexpression lines with high expression level of Lsa-miR396a showed smaller leaves, the opposite phenotype of LsaGRF5-OE (Fig. 6C). We detected the expression level of LsaGRF5 in Lsa-miR396$\mathrm{OE}$ lines, and the results revealed that they were suppressed in Lsa-miR396a-OE lines (Fig. 6D). To quantify the leaf growth changes in these transgenic lines, we measured the length and width of the outermost leaves at 10-leaf stage. The leaves in LsaGRF5 overexpression lines were significantly larger than these of YDL transformed by empty vector (OE-) both in length and width (Fig. 6E and Table S2). However, the leaf length and width of $L s a-$ miR396a overexpression lines were significantly smaller than these of wide type 'YDL' (Fig. 6E and Table S2). These results suggested that $L s a G R F 5$ was identified as a regulatory factor in leaf size, while Lsa-miR396a was also found to function in leaf size by the regulation of expression of LsaGRF5.

\section{Discussion}

GROWTH-REGULATING FACTOR, a type of plant specific transcription factor, plays important roles in plant growth and development. Our studies focus on the genome organization, conservation and function of GRFs in lettuce. 15 GRF genes were identified based on the recently released genome data of lettuce cultivar 'Salinas' [39]. The regulatory genes are considered to be preferentially retained after genome duplications [41]. The GRF gene family has subjected to two expansions with one occurred through the whole-genome triplication in the common ancestor of eudicots and the other one occurred during independent whole-genome duplications in various plants [8]. Among the 15 identified LsaGRFs, not all LsaGRF genes contained both QLQ and WRC domains. LsaGRF7 contains only WRC domain, and LsaGRF4 contains two WRC domains but no QLQ domain, which might be the results from whole-genome duplication and recombination, and might play distinct roles in plant growth regulation (Fig. 2B). Interestingly, LsaGRF9, like rice and maize GRF10, had truncated C-terminal [14, 16, 42]. Overexpression of ZmGRF10 may break the homeostasis of GRF/GIF (GRF INTERACTION FACTOR) to affect leaf growth, whether or not 


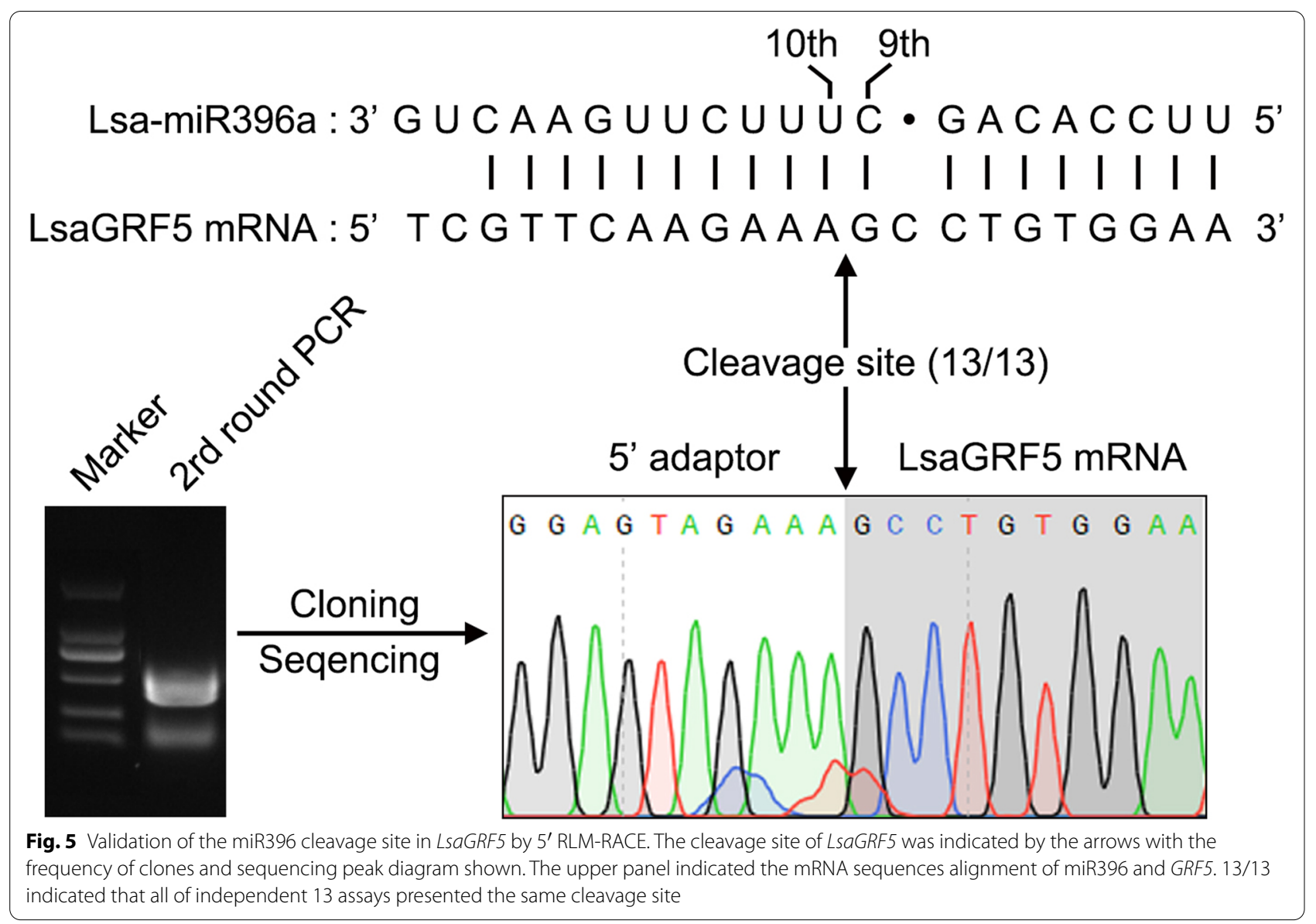

LsaGRF9 functioned in the same way needs to be further addressed [42].

Through subcellular location and transactivation assay, LsaGRF5 probably worked as transcriptional factor in cell nucleus. Notably, AtGRF5 was proved to be able to interact with GIF1 to regulate cell proliferation in leaf primordium [3]. In addition, GIF proteins have recently been reported to play a role in transcription regulation not only by the interactions with GRFs but also with various chromatin remodeling proteins [12, 43]. Whether there were functional GIF genes in lettuce, and if LsaGRF5 could interact with GIF to regulate leaf growth needs further investigation. It is already known that miR396 directly cleaves the GRF genes on their complementary sequence to suppress their expression [30]. In this study, we firstly predicted that there were $14 G R F$ genes, except for $L s a G R F 9$, as the putative targets of Lsa-miR396 based on the free energies of duplex structures analysis. The cleavage sites of LsaGRF5 were confirmed by $5^{\prime}$ RACE in vivo. At the same time, the expression of LsaGRF5 was significantly decreased in Lsa-miR396a-OE lines. Among nine GRF genes in Arabidopsis, AtGRF5 and AtGRF6 were found not to be the target of miR396 [29, 30].
AtGRF5, AtGRF6 and LsaGRF5 belonged to group VII, while LsaGRF9 was in group III (Fig. 3), suggesting that the regulation pattern of miR396-GRFs might be distinct in lettuce and Arabidopsis thaliana. Recently, AtGRF5 was found to function in chloroplast development, nitrogen signaling and senescence, besides leaf development [44]. Therefore, besides some conserved function characteristic of GRF genes, it is valuable to know whether $L s a$ GRF5 has other functions.

Expression profile of genes would help us to predict their potential biological function. The expression patterns of GRFs have been previously investigated. They usually express in growing zones of roots and shoots where cell proliferation occurs $[2,3,10,11,13,15]$. Here, we detected the expression of LsaGRF5 in root, stem, cotyledon, leaf, bud and mature flower, and revealed that LsaGRF5 was highly expressed in bud where cell proliferation occurs violently. Moreover, expression level of $A t G R F$ is suppressed during plant aging [2,30]. The expression of LsaGRF5 in bud was significantly higher than that in mature flower, which is consistent with previous results that GRFs functioned in the early stages of the growth and development in different tissues [2, 


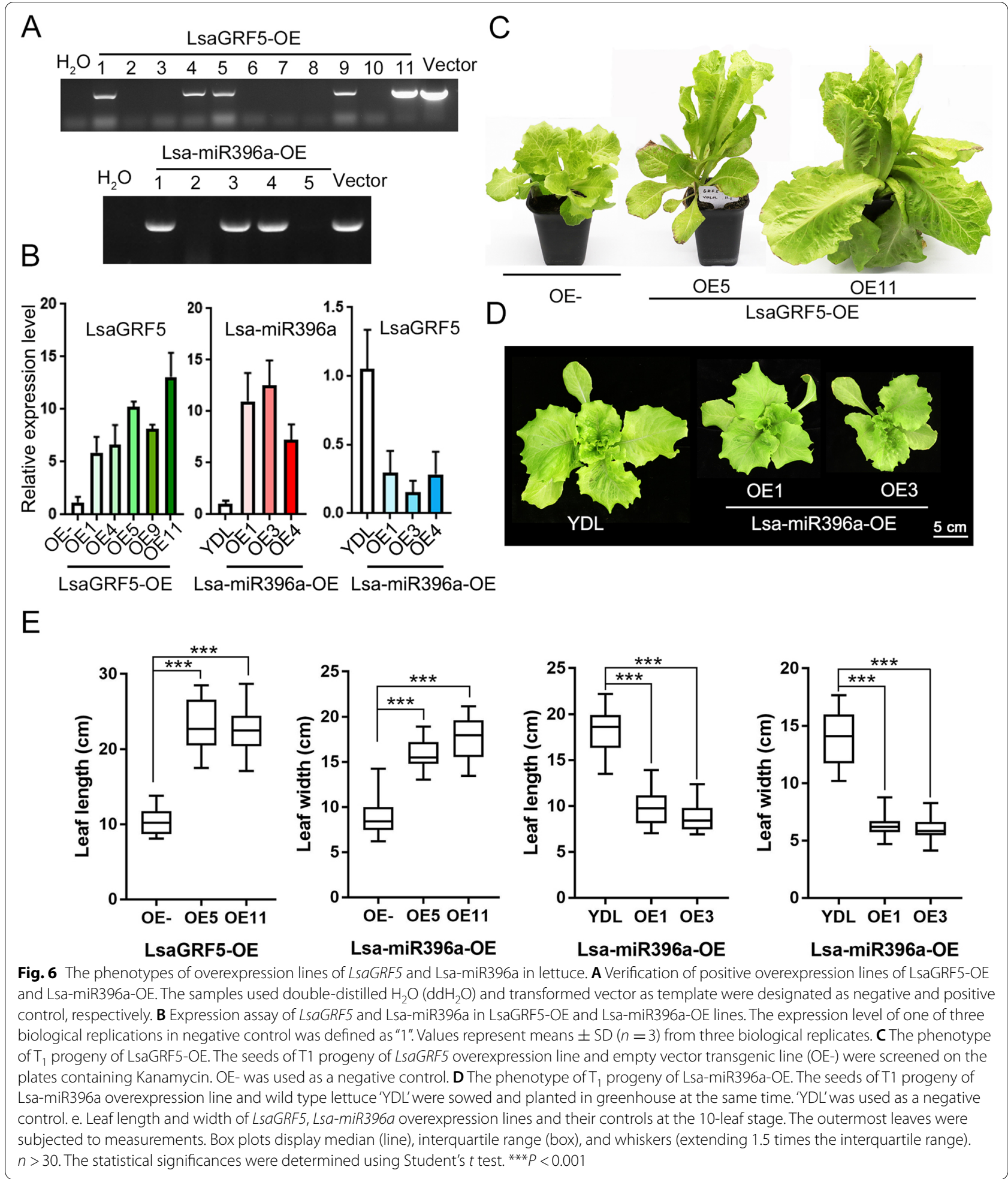

$3,30]$. In previous researches, the strong expressions of all AtGRF genes were found in the shoot apical region and flower buds, where no morphological changes were observed [2]. Instead, the alterations in leaf growth and development were detected, though the expression levels of $A t G R F$ genes were very low in leaves [2]. These results were consistent with these in this study. All AtGRF genes have low expression level in leaf and high in root, bud and 
mature flower, which is consistent with our expression results of LsaGRF5 in our manuscript [2]. The expression patterns of other $L s a G R F$ genes should be detected in future work. The overexpressed AtGRF5 could increase the leaf area by increasing the cell number, but not cell area [3]. The LsaGRF5 overexpression lines also exhibited the larger leaves, which caused by increased cell number or area should be deeply investigated.

\section{Conclusion}

In this study, we firstly identified all of the GRF gene family members in lettuce. The phylogenic relationship of these GRFs genes in lettuce with their counterparts in Arabidopsis thaliana and rice and conserved motif were evaluated. GRFs were well-known as target genes of miR396s. Therefore, thus is a daily use, we also characterized the chromosomal location of the stemloop sequences features of Lsa-miR396s. Furthermore, LsaGRF5 could probably function as a transcriptional factor in cell nucleus through subcellular location observation and transactivation assay. Overexpression of $L s a-$ GRF5 could stimulate the leaf growth leading to bigger leaves, while overexpression of Lsa-miR396a exhibited smaller leaves with suppressed expression of LsaGRF5. In summary, the expression of LsaGRF5 was regulated by Lsa-miR396 through the cleavage of complementary sequences to control leaf growth. Our findings will facilitate further understanding of the functions of GRF genes and help elucidating the leaf development mechanism in lettuce.

\section{Methods}

\section{Plant materials and growth conditions}

The lettuce (Lactuca sativa L.) cultivar of Romaine type, cv. 'YIDALI' (YDL), was used for transformation in this study. 'YIDALI' (YDL) was a commercial variety cultivated by Beijing Vegetable Research Center and the seeds were also provided by Beijing Vegetable Research Center, Beijing Academy of Agriculture and Forestry Science, Beijing, China. The sterilized lettuce seeds were grown on Murashige and Skoog (MS) medium plus 3\% sucrose and $0.6 \%$ agar $(\mathrm{pH} 5.8)$ at $25^{\circ} \mathrm{C}$ in a 16 -h-light 18-h-dark cycle. The full expanded cotyledons were used for transformation. The transgenic lettuce plants were grown in a growth chamber under a photoperiod of 16 -h light $\left(200 \mu \mathrm{mol} \mathrm{m}^{-2} \mathrm{~s}^{-1}\right)$ and 8 -h dark at $25^{\circ} \mathrm{C}$. When the fifth true leaf was fully expanded, the lettuce plants were transplanted into a greenhouse in Beijing Vegetable Research Center under standard greenhouse conditions.

For lettuce transgenic lines, we collected the seeds of T1 progeny from T0 seedlings, which were grown in a growth chamber described above. The T1 seeds of GRF5 overexpression lines were screened on the plate containing Kanamycin. The T1 generation of transgenic lines transformed with the empty vector (OE-) were used as the control and the seeds of it were screened simultaneously. And then the seedlings were transferred to the same size pots for genotyping. The T1 seeds of Lsa-miR396a overexpression lines and wide-type control 'YDL' were sown directly in soil in the greenhouse described above without antibiotic screening and then we detected the transformation positive lines using genomic PCR and qRT-PCR. The phenotypes of transgenic positive and wide-type lines were observed in greenhouse.

\section{Identification of GRF genes in lettuce}

The genome sequences of lettuce (Lactuca sativa V8) were downloaded from the Phytozome (https://phyto zome.jgi.doe.gov/pz/portal.html). The sequences of Lsa-miR396s were obtained from PmiREN (Plant miRNA Encyclopedia, http://www.pmiren.com) [45]. The sequences of AtGRFs and OsGRFs were retrieved from the Arabidopsis thaliana Information Resource (http://www.arabidopsis.org/) and China Rice Data Centre (http://www.ricedata.cn/) respectively. The amino acid and nucleotide sequences of AtGRFs and OsGRFs were used for BLASTP and BLASTN $(\mathrm{E}<0.01)$ searching in Phytozome (Lactuca sativa V8) to obtain a list of putative $L s a G R F$ genes. Subsequently, the amino acid sequences of the obtained putative LsaGRFs were reiteratively used for BLAST searching. The conserved protein domains in the GRF proteins, including QLQ (PF08880) and WRC (PF08879), were used for searching in the lettuce Protein Database in GRAMENE [46], and the protein domains of LsaGRFs were confirmed in the Pfam database (http://pfam.sanger.ac.uk/)(E-value $<1 \times 10^{-4}$ ) [47]. Finally, the results were supplemented using the HMMER software.

\section{Characterization of LsaGRFs and miR396s}

The molecular masses of the putative GRF proteins were calculated using the Compute $\mathrm{pI} / \mathrm{Mw}$ tool of ExPaSy (http://web.expasy.org/compute_pi/). Schematic Lsa$G R F$ gene structure diagrams were drawn using the Gene Structure Display Server (http://gsds.cbi.pku.edu.cn/). Protein sequence motifs were predicted using the MEME program (http://meme.sdsc.edu/meme/). The physical position of each LsaGRF gene on the ten Chinese cabbage chromosomes was determined from the Chinese cabbage database (BRAD, http://Chinesecabbagedb.org/ $\mathrm{brad} /$ ) and marked on each chromosome using the MapInspect software (http://mapinspect.software.informer. com). The combination of phylogenetic tree, gene, and protein structures was generated using the iTOL tool (http://itol.embl.de) [48]. 


\section{Phylogenetic analysis}

To construct the phylogeny of the GRFs from various species, multiple sequence alignments for all GRF amino acid sequences were conducted using MEGA 7.0 with default settings [49]. Phylogenetic analyses were carried out with a Neighbor-Joining method using MEGA 7.0 [49].

\section{Lettuce protoplast isolation and subcellular location of LsaGRF5}

The coding sequence of $L s a G R F 5$ was cloned into the pSAT6-EGFP-N1 vector. The 35S:LsaGRF5-GFP and 35S:GFP plasmids $(1 \mu \mathrm{g} / \mu \mathrm{L}, 5 \mu \mathrm{L}$ each) were transformed into protoplasts of the lettuce 'YDL' by means of polyethylene glycol treatment [50]. Transformed protoplasts were observed using a fluorescence microscope (Leica TCS SP5). Images were analyzed with Image LASAF software. Ghd7-mCherry was used as controls for nuclear [51]. Primers used were listed in Table S3.

\section{Transactivation assay based on the yeast GAL4 system}

The transcriptional activity of LsaGRF5 was evaluated in yeast cells. The full-length coding sequence, $\mathrm{N}$-terminal LsaGRF5 DNA-binding domain (1-154 aa) and the C-terminal putative activation domain (155-317 aa) were (respectively) cloned into pBD-GAL4vector. The empty vectors pGBKT7 and GAL4 were used as negative and positive controls, respectively. All of these constructs were individually introduced into cells of yeast strain Y2HGold containing the AUR1-C and MEL1 reporter genes. Yeast cell transformation was carried out using the instructions in the Yeast maker ${ }^{\mathrm{TM}}$ Yeast Transformation System 2 User Manual. The yeast transformants were grown on $\mathrm{SD} /-\operatorname{Trp}$ and $\mathrm{SD} /-\operatorname{Trp} /$ in the presence of Aba and X-alpha-gal plates for $2-4 \mathrm{~d}$ at $30^{\circ} \mathrm{C}$ to identify transactivation activity (Yeast Protocols Handbook; Clontech, Mountain View, CA, USA). Primers used were showed in Table S3.

\section{RNA extraction and qRT-PCR}

Total RNA was extracted from the leaves using a plant RNeasy kit (Tiangen, Beijing, China). RNA was reverse transcribed into cDNA with a PrimeScript ${ }^{\mathrm{TM}} \mathrm{RT}$ reagent Kit (Takara, Osaka, Japan). Real-time PCR reactions were performed using the SYBR Green I Master Mix and were quantified in a Light Cycler 480 II instrument (Roche, Basel, Switzerland). The PCR program comprised an initial step at $94^{\circ} \mathrm{C}$ for $30 \mathrm{~s}$, followed by 40 cycles of $94^{\circ} \mathrm{C}$ for $10 \mathrm{~s}$ and $58^{\circ} \mathrm{C}$ for $30 \mathrm{~s}$. Amplification was followed by heating for $1 \mathrm{~min}$ at $60-95^{\circ} \mathrm{C}$ for melting curve analysis. Each reaction was performed with three replications using $5 \mu \mathrm{L}$ of Master Mix, $0.25 \mu \mathrm{M}$ of each primer, $1 \mu \mathrm{L}$ of diluted cDNA, and DNase-free water to a final volume of $10 \mu \mathrm{L}$. Three biological replicates were collected for each sample. According to Yu et al. 2020 [52], the lettuce actin genes were used as internal controls to normalize the transcript levels of target genes. Relative gene quantification was calculated by the comparative $\Delta \Delta$ Ct method [53]. The average $2-\Delta \Delta \mathrm{Ct}$ values were used to determine differences in gene transcript levels. The lengths of PCR products were among 300 to $500 \mathrm{bp}$. The PCR products were sequenced in a commercial DNA sequencing company. The primers were designed using the Primer Premier 6.0 software and were shown in Table S3.

\section{Plasmid construction and plant transformation}

The cloning primers of LsaGRF5 and Lsa-MIR396a were designed based on the genome sequences of lettuce ( $L a c$ tuca sativa V8) from the Phytozome (https://phytozome. jgi.doe.gov/pz/portal.html). The coding sequences of LsaGRF5 and Lsa-MIR396a were cloned from the lettuce 'YDL' and constructed into the overexpression vector pEZR(K)-LC [54] driven by $35 \mathrm{~S}$ promoter using $2 \mathrm{X}$ Seamless Cloning Mix (Biomed, CL117-01, Beijing). The primers used for vector construction were listed in Table S3.

The recombinant constructs were transformed into Lettuce using Agrobacterium-mediated transformation following the leaf disk method [55]. The seeds sterilized with $10 \%$ bleach were sowed on Murashige and Skoog MS media at $22^{\circ} \mathrm{C}, 16$-h-light $/ 8$-h-dark, in a growth chamber. After 5 days, cotyledons were cut and immersed in a suspension of Agrobacterium $\left(\mathrm{OD}_{600}=0.6\right)$ for $15 \mathrm{~min}$ and then were transferred to the co-cultivation media (MS with $1 \mathrm{mM}$ acetosyringone) in the dark for 2 days. The cocultivated cotyledons were subjected to the selection and shoot-inducing media (1x MS, $0.1 \mathrm{mg} / \mathrm{L}$ 1-naphthlcetic acid, $0.1 \mathrm{mg} / \mathrm{L}$ 6-BA, $50 \mathrm{mg} / \mathrm{L}$ Kan, $300 \mathrm{mg} / \mathrm{L}$ Timentin) at $22^{\circ} \mathrm{C}$ for about 2 weeks. The young buds were transferred to the root-inducing media (MS, $300 \mathrm{mg} / \mathrm{L}$ Timentin). Finally, the resistant seedlings were transferred to soil and further verified by PCR and qRT-PCR.

\section{MiR396 cleavage site analysis}

A $5^{\prime}$ RLM-RACE was used to detect the miR396 cleavage site in miR396 target genes and was performed accordingly [56]. Total RNA of 'YDL' leaves was extracted using a plant RNeasy kit (Tiangen, Beijing, China). Then $5^{\prime}$ adaptor ligation RNA was prepared according to the kit (NEB, M0204, MA, USA). The fist chain cDNA was synthesized based on PrimeScript ${ }^{\mathrm{TM}}$ RT reagent Kit kit (Takara, RR047, Osaka, Japan). Furtherly, the RLM-RACE reactions were amplified with the enzyme TKS (Takara, Cat\# AI51320A, Osaka, Japan) and 2 X TransStart FastPfu PCR SuperMix (TransGene, Cat\# AS221-01, Beijing, 
China), respectively. Finally, the target DNA fragment was purified and cloned into vector using pEASY-Blunt Zero Cloning Kit (TransGene, CB501-01, Beijing, China). At least 15 positive clones of each gene were picked for sequencing. The gene-specific primers (GSPs) and 5'adaptor sequences were listed in Table S3.

\section{Abbreviations}

MW: Molecular weight; GRFs: GROWTH-REGULATING FACTORs; GA: Gibberellic acid; TF: Transcription factor; SAM: Shoot apical meristems; Ad-Ab: Adaxialabaxial; GIFs: GRF-Interacting Factors; AbA: Aureobasidin A; MS: Murashige and Skoog; pl: Isoelectric point; qRT-PCR: Quantitative real-time polymerase chain reaction; 5' RLM RACE assay: 5' RNA ligase-mediated rapid amplification of CDNA ends assay.

\section{Supplementary Information}

The online version contains supplementary material available at https://doi. org/10.1186/s12870-021-03261-6.

Additional file 1: Figure S1. The amino acid sequences alignment of LsaGRF genes. Figure S2. Phylogenetic analysis of Lsa-miR396s. Figure S3. The degree of Lsa-miR396a complementarity to all LsaGRFs. Figure S4. The phenotypes of overexpression lines of LsaGRF5 in Arabidopsis

Additional file 2: Table S1. Number of GRF genes and miR396s have been identified in different species

Additional file 3: Table S2. The length and width of $\angle S A G R F 5-O E$ and Lsa-miR396a-OE leaves

Additional file 4: Table S3. The sequences of Primers used in this study

Additional file 5. The original gel images used in this article

\section{Acknowledgements}

We would like to thank Dr. Maoyin Li for his critical reading on the manuscript.

\section{Plant ethics}

Experimental materials were obtained from Beijing Vegetable Research Center and we have got the permission to use. Experimental research and field studies comply with Beijing Vegetable Research Center guidelines. The wide-type lettuce used in this study was a commercial variety cultivated by the Beijing Vegetable Research Center and was not deposited in a publicly available herbarium.

\section{Authors' contributions}

D. L, D. Z., and B. Z. designed experiments. B. Z., Y. T., Z. S. and D. Z. identified and characterized the GRF genes family in lettuce. B. Z., Y.T., K. L., Z. Z., and X. L. carried out the experiment. B. Z., and D. L. wrote the paper. All authors discussed the results and commented on the manuscript. The author(s) read and approved the final manuscript.

\section{Funding}

This work was supported by grants from the Innovation and Development Programs of Beijing Vegetable Research Center [KYCX202002-01 and KYCX201901-01], the Beijing Academy of Agriculture and Forestry Science [KJCX20200113].

\section{Availability of data and materials}

The genome and protein sequences of lettuce (Lactuca sativa V8) were downloaded from the Phytozome (https://phytozome.jgi.doe.gov/pz/portal.html). The sequences of Lsa-miR396s were obtained from PmiREN (Plant miRNA Encyclopedia, http://www.pmiren.com). All of the datasets supporting the results of this article are included within the article and its additional files. The sequences of AtGRFs and OSGRFs were retrieved from the Arabidopsis thaliana Information Resource (http://www.arabidopsis.org/) and China Rice Data Centre (http://www.ricedata.cn/) respectively. The sequences, alignments and phylogeny data were uploaded to the TreeBASE repository (https://www.treeb ase.org/). The accession number is 28704 and the data could be checked on the website: http://purl.org/phylo/treebase/phylows/study/TB2:S28704.

\section{Declarations}

Ethics approval and consent to participate

Not applicable.

\section{Consent for publication}

Not applicable.

\section{Competing interests}

The authors declare that they have no competing interests.

\section{Author details}

${ }^{1}$ National Engineering Research Center for Vegetables, Beijing Vegetable Research Center, Beijing Academy of Agriculture and Forestry Science, Beijing 100097, PR China. ${ }^{2}$ Beijing Key Laboratory of Vegetable Germplasm Improvement, Beijing 100097, PR China. ${ }^{3}$ Key Laboratory of Biology and Genetic Improvement of Horticultural Crops (North China), Ministry of Agriculture and Rural Affairs of the P. R. China, Beijing 100097, PR China. ${ }^{4}$ Biotechnology Research Center, China Three Gorges University, Yichang 443002, PR China. ${ }^{5}$ College of Life Sciences, Shandong Normal University, Jinan 250014, PR China. ${ }^{6}$ CAS Center for Excellence in Molecular Plant Sciences, Shanghai Institute of Plant Physiology and Ecology, Chinese Academy of Sciences, Shanghai 200032, PR China.

Received: 2 March 2021 Accepted: 6 October 2021

Published online: 23 October 2021

\section{References}

1. van der Knaap E, Kim JH, Kende H. A novel gibberellin-induced gene from rice and its potential regulatory role in stem growth. Plant Physiol. 2000;122(3):695-704

2. Kim JH, Choi D, Kende $\mathrm{H}$. The AtGRF family of putative transcription factors is involved in leaf and cotyledon growth in Arabidopsis. Plant J. 2003;36(1):94-104

3. Horiguchi G, Kim GT, Tsukaya H. The transcription factor AtGRF5 and the transcription coactivator AN3 regulate cell proliferation in leaf primordia of Arabidopsis thaliana. Plant J. 2005;43(1):68-78.

4. Kim JH, Lee BH. GROWTH-REGULATING FACTOR4 of Arabidopsis thaliana is required for development of leaves, cotyledons, and shoot apical meristem. J Plant Biol. 2006:49(6):463-8.

5. Powell Anahid E, Lenhard M. Control of organ size in plants. Curr Biol. 2012;22(9):R360-7.

6. Hepworth J, Lenhard M. Regulation of plant lateral-organ growth by modulating cell number and size. Curr Opin Plant Biol. 2014;17:36-42.

7. Kim JH, Tsukaya H. Regulation of plant growth and development by the GROWTH-REGULATING FACTOR and GRF-INTERACTING FACTOR duo. J Exp Bot. 2015;66(20):6093-107.

8. Omidbakhshfard MA, Proost S, Fujikura U, Mueller-Roeber B. Growth-regulating factors (GRFs): a small transcription factor family with important functions in plant biology. Mol Plant. 2015;8(7):998-1010.

9. Hewezi T, Maier TR, Nettleton D, Baum TJ. The Arabidopsis MicroRNA396GRF1/GRF3 regulatory module acts as a developmental regulator in the reprogramming of root cells during cyst nematode infection. Plant Physiol. 2012;159(1):321.

10. Kim J-S, Mizoi J, Kidokoro S, Maruyama K, Nakajima J, Nakashima K, et al. Arabidopsis growth-regulating factor7 functions as a transcriptional repressor of abscisic acid- and osmotic stress-responsive genes, including DREB2A. Plant Cell. 2012;24(8):3393-405.

11. Bao M, Bian H, Zha Y, Li F, Sun Y, Bai B, et al. Han N: miR396a-mediated basic Helix-loop-Helix transcription factor bHLH74 repression acts as a regulator for root growth in Arabidopsis seedlings. Plant Cell Physiol. 2014;55(7):1343-53.

12. Debernardi JM, Mecchia MA, Vercruyssen L, Smaczniak C, Kaufmann K, Inze $D$, et al. Post-transcriptional control of GRF transcription factors by 
microRNA miR396 and GIF co-activator affects leaf size and longevity. Plant J. 2014;79(3):413-26.

13. Liang G, He H, Li Y, Wang F, Yu D. Molecular mechanism of microRNA396 mediating pistil development in Arabidopsis. Plant Physiol. 2014;164(1):249.

14. Liu H, Guo S, XuY, Li C, Zhang Z, Zhang D, et al. OsmiR396d-regulated OsGRFs function in floral organogenesis in rice through binding to their targets OsJMJ706 and OsCR4. Plant Physiol. 2014;165(1):160.

15. Pajoro A, Madrigal P, Muiño JM, Matus JT, Jin J, Mecchia MA, et al. Dynamics of chromatin accessibility and gene regulation by MADS-domain transcription factors in flower development. Genome Biol. 2014;15(3):R41.

16. Choi D, Kim JH, Kende H. Whole genome analysis of the OsGRF gene family encoding plant-specific putative transcription activators in rice (Oryza sativa L.). Plant Cell Physiol. 2004:45(7):897-904.

17. Treich I, Cairns BR, de los Santos T, Brewster E, Carlson M. SNF11, a new component of the yeast SNF-SWI complex that interacts with a conserved region of SNF2. Mol Cell Biol. 1995;15(8):4240.

18. Raventós D, Skriver K, Schlein M, Karnahl K, Rogers SW, Rogers JC, et al. HRT, a novel zinc finger, transcriptional repressor from barley. J Biol Chem. 1998;273(36):23313-20.

19. Liu J, Hua W, Yang HL, Zhan GM, Li RJ, Deng LB, et al. The BnGRF2 gene (GRF2-like gene from Brassica napus) enhances seed oil production through regulating cell number and plant photosynthesis. J Exp Bot. 2012;63(10):3727-40.

20. Hu J, Wang $Y$, Fang $Y$, Zeng $L, X u J, Y u ~ H$, et al. A rare allele of GS2 enhances grain size and grain yield in rice. Mol Plant. 2015;8(10):1455-65.

21. Sun $P$, Zhang $W$, Wang $Y$, He Q, Shu F, Liu H, et al. OsGRF4 controls grain shape, panicle length and seed shattering in rice. J Integr Plant Biol. 2016;58(10):836-47.

22. Kim JH. Biological roles and an evolutionary sketch of the GRF-GIF transcriptional complex in plants. BMB Rep. 2019:52(4):227-38.

23. Luo G, Palmgren M. GRF-GIF chimeras boost plant regeneration. Trends Plant Sci. 2020;26(3):201-4

24. Arvidsson S, Pérez-Rodríguez P, Mueller-Roeber B. A growth phenotyping pipeline for Arabidopsis thaliana integrating image analysis and rosette area modeling for robust quantification of genotype effects. New Phytol. 2011;191(3):895-907.

25. Bartel DP. MicroRNAs: genomics, biogenesis, mechanism, and function. Cell. 2004;116(2):281-97.

26. Zhang B, Stellwag EJ, Pan X. Large-scale genome analysis reveals unique features of microRNAs. Gene. 2009:443(1):100-9.

27. Mallory AC, Bouché N. MicroRNA-directed regulation: to cleave or not to cleave. Trends Plant Sci. 2008;13(7):359-67.

28. Debernardi JM, Rodriguez RE, Mecchia MA, Palatnik JF. Functional specialization of the plant miR396 regulatory network through distinct microRNA-target interactions. PLoS Genet. 2012;8(1):e1002419.

29. Liu D, Song Y, Chen Z, Yu D. Ectopic expression of miR396 suppresses GRF target gene expression and alters leaf growth in Arabidopsis. Physiol Plant. 2009;136(2):223-36

30. Rodriguez RE, Mecchia MA, Debernardi JM, Schommer C, Weigel D, Palatnik JF. Control of cell proliferation in Arabidopsis thaliana by microRNA miR396. Development. 2010;137(1):103.

31. Wang L, Gu X, Xu D, Wang W, Wang H, Zeng M, et al. miR396-targeted AtGRF transcription factors are required for coordination of cell division and differentiation during leaf development in Arabidopsis. J Exp Bot. 2011;62(2):761-73.

32. Zhang DF, Li B, Jia GQ, Zhang TF, Dai JR, Li JS, et al. Isolation and characterization of genes encoding GRF transcription factors and GIF transcriptional coactivators in maize (Zea mays L.). Plant Sci. 2008;175(6):809-17.

33. Wang F, Qiu N, Ding Q, Li J, Zhang Y, Li H, et al. Genome-wide identification and analysis of the growth-regulating factor family in Chinese cabbage (Brassica rapa L. ssp. pekinensis). BMC Genomics. 2014;15(1):807.

34. Cao D, Wang J, Ju Z, Liu Q, Li S, Tian H, et al. Regulations on growth and development in tomato cotyledon, flower and fruit via destruction of miR396 with short tandem target mimic. Plant Sci. 2016;247:1-12.

35. Cao Y, Han Y, Jin Q, Lin Y, Cai Y. Comparative genomic analysis of the GRF genes in Chinese pear (Pyrus bretschneideri Rehd), poplar (populous), grape (Vitis vinifera), Arabidopsis and Rice (Oryza sativa). Front Plant Sci. 2016;7:1750.
36. Ma JQ, Jian HJ, Yang B, Lu K, Zhang AX, Liu P, et al. Genome-wide analysis and expression profiling of the GRF gene family in oilseed rape (Brassica napus L.). Gene. 2017:620:36-45.

37. Zhang J, Li Z, Jin J, Xie X, Zhang H, Chen Q, et al. Genome-wide identification and analysis of the growth-regulating factor family in tobacco (Nicotiana tabacum). Gene. 2018;639:117-27.

38. Wang J, Zhou H, Zhao Y, Sun P, Tang F, Song $X$, et al. Characterization of poplar growth-regulating factors and analysis of their function in leaf size control. BMC Plant Biol. 2020;20(1):509.

39. Reyes-Chin-Wo S, Wang Z, Yang X, Kozik A, Arikit S, Song C, et al. Genome assembly with in vitro proximity ligation data and whole-genome triplication in lettuce. Nat Commun. 2017:8:14953.

40. Yang C, Li D, Liu X, Ji C, Hao L, Zhao X, et al. OsMYB103L, an R2R3-MYB transcription factor, influences leaf rolling and mechanical strength in rice (Oryza sativa L.). BMC Plant Biol. 2014;14:158.

41. Maere S, De Bodt S, Raes J, Casneuf T, Van Montagu M, Kuiper M, et al. Modeling gene and genome duplications in eukaryotes. Proc Natl Acad Sci U S A. 2005:102(15):5454.

42. Wu L, Zhang D, Xue M, Qian J, He Y, Wang S. Overexpression of the maize GRF10, an endogenous truncated growth-regulating factor protein, leads to reduction in leaf size and plant height. J Integr Plant Biol. 2014;56(11):1053-63.

43. Vercruyssen L, Verkest A, Gonzalez N, Heyndrickx KS, Eeckhout D, Han S-K, et al. ANGUSTIFOLIA3 binds to SWI/SNF chromatin remodeling complexes to regulate transcription during Arabidopsis leaf development. Plant Cell. 2014;26(1):210-29.

44. Vercruyssen L, Tognetti VB, Gonzalez N, Van Dingenen J, De Milde L, Bielach A, et al. GROWTH REGULATING FACTOR5 stimulates Arabidopsis chloroplast division, photosynthesis, and leaf longevity. Plant Physiol. 2015;167(3):817.

45. Guo Z, Kuang Z, Wang Y, Zhao Y, Tao Y, Cheng C, et al. PmiREN: a comprehensive encyclopedia of plant miRNAs. Nucleic Acids Res. 2020:48(D1):D1114-21.

46. Ware DH, Jaiswal P, Ni J, Yap IV, Pan X, Clark KY, et al. Gramene, a tool for grass genomics. Plant Physiol. 2002;130(4):1606.

47. Punta M, Coggill PC, Eberhardt RY, Mistry J, Tate J, Boursnell C, et al. The Pfam protein families database. Nucleic Acids Res. 2012;40:D290-301.

48. Letunic I, Bork P. Interactive Tree Of Life v2: online annotation and display of phylogenetic trees made easy. Nucleic Acids Res. 2011;39:W475-8.

49. Kumar S, Stecher G, Tamura K. MEGA7: molecular evolutionary genetics analysis version 7.0 for bigger datasets. Mol Biol Evol. 2016;33(7):1870-4.

50. Yoo SD, Cho YH, Sheen J. Arabidopsis mesophyll protoplasts: a versatile cell system for transient gene expression analysis. Nat Protoc. 2007;2(7):1565-72.

51. Xue $W$, Xing $Y$, Weng $X$, Zhao $Y$, Tang $W$, Wang $L$, et al. Natural variation in Ghd7 is an important regulator of heading date and yield potential in rice. Nat Genet. 2008:40(6):761-7.

52. Yu C, Yan C, Liu Y, Liu Y, Jia Y, Lavelle D, et al. Upregulation of a KN1 homolog by transposon insertion promotes leafy head development in lettuce. Proc Natl Acad Sci. 2020;1 17(52):33668.

53. Schmittgen TD, Livak KJ. Analyzing real-time PCR data by the comparative CT method. Nat Protoc. 2008;3(6):1101-8.

54. Brown BA, Cloix C, Jiang GH, Kaiserli E, Herzyk P, Kliebenstein DJ, et al. A UV-B-specific signaling component orchestrates plant UV protection. Proc Natl Acad Sci U S A. 2005;102(50):18225.

55. Michelmore R, Marsh E, Seely S, Landry B. Transformation of lettuce (Lactuca sativa) mediated by Agrobacterium tumefaciens. Plant Cell Rep. 1987;6(6):439-42

56. Wang C, Fang J. RLM-RACE, PPM-RACE, and qRT-PCR: an integrated strategy to accurately validate mirna target genes. In: Rederstorff M, editor. Small Non-Coding RNAs: Methods and Protocols. New York: Springer New York; 2015. p. 175-86.

\section{Publisher's Note}

Springer Nature remains neutral with regard to jurisdictional claims in published maps and institutional affiliations. 